\title{
On Total Domination Polynomials of Certain Graphs
}

\author{
S. Sanal ${ }^{1}$ and H. E. Vatsalya ${ }^{2}$ \\ 1. Faculty of Mathematics, Ibri College of Technology, Sultanate of Oman. \\ 2. Faculty of Mathematics, Ibri College of Technology, Sultanate of Oman.
}

Received: December 14, 2014 / Accepted: January 11, 2015 / Published: March 25, 2016.

\begin{abstract}
We have introduced the total domination polynomial for any simple non isolated graph $G$ in [7] and is defined by $\mathrm{D}_{t}(G, x)=$ $\sum_{i=\gamma_{t}(\boldsymbol{G})}^{n} \boldsymbol{d}_{t}(G, i) x^{i}$, where $\mathrm{d}_{t}(G, i)$ is the cardinality of total dominating sets of $G$ of size $i$, and $\gamma_{t}(G)$ is the total domination number of $G$. In [7] We have obtained some properties of $\mathrm{D}_{\mathrm{t}}(G, x)$ and its coefficients. Also, we have calculated the total domination polynomials of complete graph, complete bipartite graph, join of two graphs and a graph consisting of disjoint components. In this paper, we presented for any two isomorphic graphs the total domination polynomials are same, but the converse is not true. Also, we proved that for any vertex transitive graph of order $n$ and for any $v \in V(G), \mathrm{d}_{t}(G, i)=\frac{\boldsymbol{n}}{\boldsymbol{i}} \mathrm{d}_{t}^{(v)}(G, i), 1 \leq i \leq n$. And, for any $k$-regular graph of order $n, \mathrm{~d}_{t}(G, i)$ $=\left(\begin{array}{c}n \\ \boldsymbol{i}\end{array}\right), i>n-k$ and $\mathrm{d}_{t}(G, n-k)=\left(\begin{array}{l}\boldsymbol{n} \\ \boldsymbol{k}\end{array}\right)-n$. We have calculated the total domination polynomial of Petersen graph $\mathrm{D}_{t}(P, x)=10 x^{4}+72 x^{5}+$ $140 x^{6}+110 x^{7}+45 x^{8}+10 x^{9}+x^{10}$. Also, for any two vertices $u$ and $v$ of a $k$-regular graph $H$ with $N(u) \neq N(v)$ and if $\mathrm{D}_{t}(G, x)=\mathrm{D}_{t}(H, x)$, then $G$ is also a $k$-regular graph.
\end{abstract}

Key words: total dominating set, total domination number, total domination polynomial.

\section{Introduction}

Let $G=(V, E)$ be a graph. For any vertex $u \in V$, we define the open neighborhood of $u$ as the set $N(u)$ defined by $N(u)=\{v / u v \in E\}$ and the closed neighborhood of $u$ as the set $N[u]$ defined by $N[u]=$ $N(u) \cup\{u\}$. For a subset $S$ of $V$, the open neighborhood of $S$ is $N(S)$ which is defined as the union of $N(u)$ for all $u \in S$ and the closed neighborhood of $S$ is defined as $N(S) \cup S$. The maximum degree of the graph $G$ is denoted by $\Delta(G)$ and the minimum degree is denoted by $\delta(G)$. A set $S$ of vertices in a graph $G$ is said to be a dominating set if every vertex $u \in V$ is either an element of $S$ or is adjacent to an element of $S$. A set of vertices in a graph $G$ is said to be a total dominating set if every vertex $u \in V$ is adjacent to an element of $S$. The domination number of a graph, denoted by $\gamma(G)$, is the minimum cardinality of the dominating sets in $G$. The total domination number of a graph $G$, denoted by $\gamma_{t}(G)$, is the minimum cardinality of the total

Corresponding author: S.Sanl Kumar Ph.D., E-mail: anfigarden@yahoo.com dominating sets in $G$.

\section{Introduction to Total Domination Polynomial}

\subsection{Definition}

Let $G$ be a graph with no isolated vertices. Let $\mathfrak{D}_{t}(G$, i) be a family of total dominating sets of $G$ with cardinality $i$ and let $\mathrm{d}_{t}(G, i)=\left|\mathfrak{D}_{t}(G, i)\right|$. Then the total domination polynomial $\mathrm{D}_{t}(G, x)$ of $G$ is defined as $\mathrm{D}_{t}(G$, $x)=\sum_{i=\gamma_{t}(G)}^{n} d_{t}(G, i) x^{i}$, where $\gamma_{\mathrm{t}}(G)$ is the total domination number of $G$.

\subsection{Example}

Consider a graph $G$ with vertex set $V=\left\{v_{1}, v_{2}, v_{3}, v_{4}\right.$, $\left.v_{5}\right\}$ and edge set $E=\left\{v_{1} v_{2}, v_{2} v_{3}, v_{2} v_{5}, v_{3} v_{4}, v_{3} v_{5}, v_{4} v_{5}\right\}$. $\mathfrak{D}_{t}(G, 1)=\phi, \mathfrak{D}_{t}(G, 2)=\left\{\left\{v_{2}, v_{3}\right\},\left\{v_{2}, v_{5}\right\}\right\}, \mathfrak{D}_{t}(G, 3)$ $=\left\{\left\{v_{1}, v_{2}, v_{3}\right\},\left\{v_{2}, v_{3}, v_{4}\right\},\left\{v_{1}, v_{2}, v_{5}\right\},\left\{v_{2}, v_{3}, v_{5}\right\},\left\{v_{2}\right.\right.$, $\left.\left.v_{4}, v_{5}\right\}\right\}, \mathfrak{D}_{t}(G, 4)=\left\{\left\{v_{2}, v_{3}, v_{4}, v_{5}\right\},\left\{v_{1}, v_{2}, v_{4}, v_{5}\right\},\left\{v_{1}\right.\right.$, $\left.\left.v_{2}, v_{3}, v_{5}\right\},\left\{v_{1}, v_{2}, v_{3}, v_{4}\right\}\right\}, \mathfrak{D}_{t}(G, 5)=\left\{\left\{v_{1}, v_{2}, v_{3}, v_{4}\right.\right.$, $\left.\left.v_{5}\right\}\right\}, \mathfrak{D}_{t}\left(G,{ }_{\mathrm{i}}\right)=\phi$, for all $i>5$. Therefore, $\mathrm{d}_{t}(G, 1)=0$, $\mathrm{d}_{t}(G, 2)=\left|\mathfrak{D}_{t}(G, 2)\right|=2, \mathrm{~d}_{t}(G, 3)=\left|\mathfrak{D}_{t}(G, 3)\right|=5, \mathrm{~d}_{t}(G$, 


$$
\begin{gathered}
4)=\left|\mathfrak{D}_{t}(G, 4)\right|=4, \mathrm{~d}_{t}(G, 5)=\left|\mathfrak{D}_{t}(G, 5)\right|=1 . \\
\mathrm{D}_{t}(G, x)=\sum_{i=\gamma_{t}(G)}^{n} d_{t}(G, i) x^{i} \\
=\mathrm{d}_{t}(G, 2) x^{2}+\mathrm{d}_{t}(G, 3) x^{3}+\mathrm{d}_{t}(G, 4) x^{4}+\mathrm{d}_{t}(G, 5) x^{5} \\
=2 x^{2}+5 x^{3}+4 x^{4}+x^{5} .
\end{gathered}
$$

Hence, the total domination polynomial of the given graph $G$ is $\mathrm{D}_{t}(G, x)=2 x^{2}+5 x^{3}+4 x^{4}+x^{5}$.

\subsection{Lemma}

The degree of the total domination polynomial of any simple graph $\mathrm{G}$ without isolated vertices is the order of the graph $\mathrm{G}$.

\subsection{Lemma}

If $H$ is a sub graph of a simple graph $G$ without isolated vertices, then the degree of the total domination polynomial of $H$ is less than or equal to the degree of the total domination polynomial of $G$.

\subsection{Remark}

If $\mathrm{H}$ is a sub graph of a graph $G$ without isolated vertices, then $\mathrm{d}_{t}(H, i)$ need not be less than or equal to $\mathrm{d}_{t}(G, i)$.

Proof:

Let $\mathrm{G}$ be a path $P_{7}$ with the vertex set $\{1,2,3,4,5,6$, $7\}$ and let $H$ be the sub graph $P_{6}$ with the vertex set $\{1$, $2,3,4,5,6\}$ of $\mathrm{P}_{7}$.

We have, $\mathfrak{D}_{\mathrm{t}}\left(P_{6}, 4\right)=\{\{2,3,5,6\},\{2,3,4,5\},\{1,2$, $5,6\},\{1,2,4,5\}\}$ and $\mathfrak{D}_{\mathrm{t}}\left(P_{7}, 4\right)=\{\{2,3,5,6\},\{2,3$, $6,7\},\{1,2,5,6\}\}$. But $\mathrm{d}_{t}\left(P_{6}, 4\right)=4$ and $\mathrm{d}_{t}\left(P_{7}, 4\right)=3$.

Thus, we conclude that the remark.

\subsection{Lemma}

Let $G_{1}$ and $G_{2}$ be two graphs with vertex sets $V_{1}$ and $V_{2}$ respectively. If $G_{1}$ and $G_{2}$ are isomorphic, then the total domination polynomials of $G_{1}$ and $G_{2}$ are equal.

\section{Proof:}

Let the graph function $f$ from $G_{1}$ to $G_{2}$ be an isomorphism. Then, $\left|V_{1}\right|=\left|V_{2}\right|$. By Lemma 2.3, the degree of total domination polynomial of $G_{1}$ is equal to the degree of total domination polynomial of $G_{2}$. Next, we have to prove $\mathrm{d}_{t}\left(G_{1}, m\right)=\mathrm{d}_{t}\left(G_{2}, m\right)$. It is enough to prove $\left|\mathfrak{D}_{t}\left(G_{1}, m\right)\right|=\left|\mathfrak{D}_{t}\left(G_{2}, m\right)\right|$. Let $\mathrm{D}_{t}\left(G_{1}, m\right)=\left\{u_{1}\right.$, $\left.u_{2}, \ldots, u_{m}\right\}$ be a total dominating set of $G_{1}$ with cardinality $m$. Let $u_{\mathrm{i}}$ and $u_{j}$ be any two adjacent vertices of $G_{1}$ in $\mathrm{D}_{t}\left(G_{1}, m\right)$. Since $G_{1}$ and $G_{2}$ are isomorphic, $f\left(u_{\mathrm{i}}\right)$ and $f\left(u_{j}\right)$ are adjacent vertices of $G_{2}$. Therefore, $\mathrm{D}_{t}\left(G_{2}\right.$, $m)=\left\{f\left(u_{1}\right), f\left(u_{2}\right), \ldots f\left(u_{m}\right)\right\}$ is a total dominating set of $G_{2}$ with cardinality $m$. Hence, $\mathrm{d}_{t}\left(G_{1}, m\right)=\mathrm{d}_{t}\left(G_{2}, m\right)$ from which it follows that the total domination polynomial of $G_{1}$ and $G_{2}$ are equal.

\subsection{Remark}

If the total domination polynomials of two graphs are equal, then the two graphs need not be isomorphic.

\section{Proof:}

We consider the following two graphs $G_{1}$ and $G_{2}$ and their total domination polynomial is $x^{3}+6 x^{4}+15 x^{5}+$ $20 x^{6}+15 x^{7}+6 x^{8}+x^{9}$.
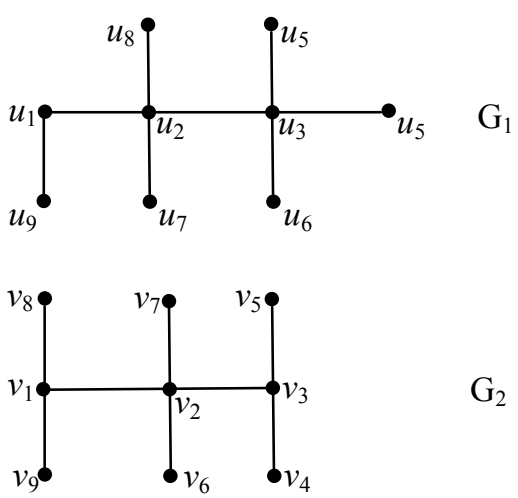

$\mathrm{G}_{2}$

But, there does not exist an isomorphism between $G_{1}$ and $G_{2}$. Hence, even if the total domination polynomials of two graphs are same, the two graphs need not be isomorphic.

\section{Total Domination Polynomial of the Petersen Graph}

\section{Definition 3.1}

A vertex - transitive graph is a graph $G$ such that for each pair of vertices $u$ and $v$ of $G$, there exists a graph automorphism $f$ on $\mathrm{G}$ such that $f(u)=v$.

Notation 3.2

Let $\mathfrak{D}_{t}^{(v)}(G, i)$ be a family of all total dominating sets of $G$ with cardinality $i$ and it contains the vertex $v$ of $G$. 
Let $\mathrm{d}_{t}^{(v)}(G, i)=\left|\mathfrak{D}_{t}^{(v)}(G, i)\right|$.

\section{Lemma 3.3}

Let $G$ be a vertex transitive graph of order $n$ and $v \in$ $V(G)$. For any $1 \leq i \leq n, \mathrm{~d}_{\mathrm{t}}(G, i)=\frac{n}{i} \mathrm{~d}_{t}^{(v)}(G, i)$.

\section{Proof:}

Let $G$ be a vertex transitive graph of order $n$. Let $D$ be a total dominating set of $G$ with cardinality $i$. Since $G$ is a vertex transitive graph, there exists a graph automorphism $f$ on $G$. Therefore, $f(D)$ is also the total dominating set of $G$ with cardinality $i$. Also, for any two vertices $u$ and $v$ of $G, \mathrm{~d}_{t}^{(u)}(G, i)=\mathrm{d}_{t}^{(v)}(G, i)$. Since $D$ is a total dominating set of $G$ with cardinality $i$, there are exactly $i$ vertices $v_{j_{1}}, v_{j_{2}}, \ldots, v_{j_{i}}$ such that $D$ counted in $d_{t}^{\left(v_{j_{r}}\right)}(G, i)$, for each $1 \leq r \leq i$. Hence, $\mathrm{d}_{t}(G, i)$ $=\frac{n}{i} \mathrm{~d}_{\mathrm{t}}^{\left({ }^{()}\right.}(G, i)$.

\section{Lemma 3.4}

Let $G$ be a $k$-regular graph of order $n$. Then, $\mathrm{d}_{\mathrm{t}}(G, i)=$ $\left(\begin{array}{l}n \\ i\end{array}\right)$ for all $i>n-k$.

\section{Proof:}

Let $G$ be a $k$-regular connected graph with vertex set $V=\left\{v_{1}, v_{2}, \ldots, v_{n}\right\}$. Since $G$ is a $k$-regular graph, each vertex of $G$ is of degree $k$. Now, remove any $k-1$ elements from $V$. Let the remaining set be $V_{1}$. All the vertices of $G$ can be dominated by the elements of $V_{1}$. Therefore, $V_{1}$ is a total dominating set of cardinality $n-$ $k+1$. Therefore, the number of total dominating sets of cardinality $n-k+1$ is the number of ways of deleting $k$-1 elements from $V$, which is $\left(\begin{array}{c}n \\ k-1\end{array}\right)$ or $\left(\begin{array}{c}n \\ n-k+1\end{array}\right)$. If we remove any $k-2$ elements from $V$, then the remaining $n$ $-(k-2)$ elements will form a total dominating set of $G$ with cardinality $n-k+2$. Therefore, $\mathrm{d}_{t}(G, n-k+2)=$ $\left(\begin{array}{c}n \\ n-k+2\end{array}\right)=\left(\begin{array}{c}n \\ k-2\end{array}\right)$. So, in general if we remove any $j$ elements from $V$ which is less than or equal to $k-1$, then the remaining elements form a total dominating set of $G$ with cardinality $n-j$ in $\left(\begin{array}{c}n \\ n-j\end{array}\right)$ ways.

Therefore, $\mathrm{d}_{t}(G, n-j)=\left(\begin{array}{c}n \\ n-j\end{array}\right)$. Hence, $\mathrm{d}_{t}(G, i)=\left(\begin{array}{c}n \\ i\end{array}\right)$ for all $i>n-k$.

\section{Lemma 3.5}

Let $G$ be a $k$ - regular graph of order $n$. Then, $\mathrm{d}_{t}(G$, $n-k)=\left(\begin{array}{l}n \\ k\end{array}\right)-n$.

\section{Proof}

Let $G$ be a $k$-regular graph with vertex set $\left\{v_{1}, v_{2}, \ldots\right.$, $\left.v_{n}\right\}$. Since $G$ is $k$ - regular, every open neighborhood of $v$ in $V$ has exactly $k$ elements. Now, for each, $v_{i} \in V$, $\mathrm{N}\left(v_{i}\right)=\left\{v_{i_{1}}, v_{i_{2}}, \ldots, v_{i_{k}}\right\}$. By removing all the elements of $\mathrm{N}\left(v_{i}\right)$ from $V, v_{i}$ becomes an isolated vertex. Therefore, $V-\mathrm{N}\left(v_{i}\right)$ is not a total dominating set. This is true for each $i=1,2, \ldots, n$. By Peigon hole principle, there are exactly $n$ non-total dominating sets of cardinality $n-k$ in $G$. Hence, by lemma $3.4 \mathrm{~d}_{t}(G, n-k)=$ $\left(\begin{array}{l}n \\ k\end{array}\right)-n$.

\section{Theorem 3.6}

The total domination polynomial of the Petersen graph $P$ is $\mathrm{D}_{\mathrm{t}}(P, x)=10 x^{4}+72 x^{5}+140 x^{6}+110 x^{7}+$ $45 x^{8}+10 x^{9}+x^{10}$

\section{Proof:}

Let $P$ be a Petersen graph of order 10 with vertex set $V=\{1,2,3,4,5,6,7,8,9,10\}$, as given in Figure.

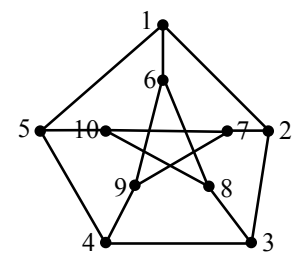

For $P$, the total domination number is $\gamma_{t}(P)=4$. First, we have to find the collection of total dominating sets of $\mathrm{P}$ of cardinality 4.

$\mathfrak{D}_{t}(P, 4)=\{\{5,7,8,10\},\{4,6,7,9\},\{3,6,8,10\}$, $\{2,7,9,10\},\{2,3,4,8\},\{1,6,8,9\},\{1,4,5,10\},\{1,2,5,6\}$. $\{1,2,3,7\},\{3,4,5,9\}\}$.

The number of total dominating sets of cardinality 4 containing one vertex labeled as ' 5 ' is 4 ,

Therefore, $\mathrm{d}_{t}(P, 4)=\mathrm{n} / 4 \cdot \mathrm{d}_{t}^{(5)}(P, 4)=\frac{10}{4} \cdot 4=10$.

Next, we shall find $\mathrm{d}_{\mathrm{t}}(P, 5)$. By Lemma 3.3, it is enough to find the total dominating sets of $P$ with cardinality 5 containing one vertex labeled as ' 6 '. 
These total dominating sets are given below:

$$
\mathfrak{D}_{t}^{(6)}(P, \quad 5)=\{\{6,7,8,9,10\}, \quad\{5,6,7,8,10\},
$$

$\{4,6,7,9,10\},\{4,6,7,8,9\},\{4,5,6,7,9\},\{3,6,8,9,10\}$, $\{3,6,7,8,10\},\{3,5,6,8,10\},\{3,4,6,8,10\},\{3,4,6,8,9\}$, $\{3,4,6,7,9\}, \quad\{3,4,5,6,9\}, \quad\{2,6,7,9,10\}, \quad\{2,4,6,7,9\}$, $\{2,3,6,8,10\},\{2,3,4,6,8\},\{1,6,8,9,10\},\{1,6,7,8,9\}$, $\{1,5,6,8,10\}, \quad[1,5,6,8,9\}, \quad\{1,4,6,8,9\}, \quad\{1,4,6,7,9\}$, $\{1,4,5,6,9\},\{1,4,5,6,10\},\{1,3,6,8,10\},\{1,3,6,8,9\}$, $\{1,2,6,8,9\}, \quad\{1,2,6,7,9\}, \quad\{1,2,5,6,10\}, \quad\{1,2,5,6,9\}$, $\{1,2,5,6,8\}, \quad\{1,2,5,6,7\}, \quad\{1,2,4,5,6\}, \quad\{1,2,3,6,8\}$, $\{1,2,3,6,7\},\{1,2,3,5,6\}\}$. Therefore, $\mathrm{d}_{t}^{(6)}(P, 5)=36$

Hence, $\mathrm{d}_{t}(P, 5)=\frac{n}{5} \cdot \mathrm{d}_{t}^{(6)}(P, 5)=\frac{10}{5} \cdot 36=72$

Next, we shall find $\mathrm{d}_{t}(P, 6)$. By Lemma 3.3, it suffices to obtain the total dominating sets of cardinality 6 containing one vertex labeled as ' 1 '. These total dominating sets are listed below:

$\begin{array}{rrr}\mathfrak{D}_{t}^{(1)}(\mathrm{P}, 6)= & \{1,6,7,8,9,10\}, & \{1,5,7,8,9,10\}, \\ \{1,5,6,8,9,10\}, & \{1,5,6,7,8,10\}, & \{1,5,6,7,8,9\}, \\ \{1,4,6,8,9,10\}, & \{1,4,6,7,9,10\}, & \{1,4,6,7,8,9\}, \\ \{1,4,5,8,9,10\}, & \{1,4,5,7,9,10\}, & \{1,4,5,7,8,10\}, \\ \{1,4,5,6,9,10\}, & \{1,4,5,6,8,10\}, & \{1,4,5,6,8,9\}, \\ \{1,4,5,6,7,10\}, & \{1,4,5,6,7,9\}, & \{1,3,6,8,9,10\}, \\ \{1,3,6,7,8,10\}, & \{1,3,6,7,8,9\}, & \{1,3,5,7,8,10\}, \\ \{1,3,5,7,8,9\}, & \{1,3,5,6,8,10\}, & \{1,3,5,6,8,9\}, \\ \{1,3,4,6,8,10\}, & \{1,3,4,6,8,9\}, & \{1,3,4,6,7,10\}, \\ \{1,3,4,6,7,9\}, & \{1,3,4,5,9,10\}, & \{1,3,4,5,8,10\}, \\ \{1,3,4,5,8,9\}, & \{1,3,4,5,7,10\}, & \{1,3,4,5,7,9\}, \\ \{1,3,4,5,6,10\}, & \{1,3,4,5,6,9\}, & \{1,2,7,8,9,10\}, \\ \{1,2,6,8,9,10\}, & \{1,2,6,7,9,10\}, & \{1,2,6,7,8,9\}, \\ \{1,2,5,7,9,10\}, & \{1,2,5,7,8,10\}, & \{1,2,5,6,9,10\}, \\ \{1,2,5,6,8,10\}, & \{1,2,5,6,8,9\}, & \{1,2,5,6,7,10\}, \\ \{1,2,5,6,7,9\}, & \{1,2,5,6,7,8\}, & \{1,2,4,8,9,10\}, \\ \{1,2,4,7,9,10\}, & \{1,2,4,6,8,9\}, & \{1,2,4,6,7,9\}, \\ \{1,2,4,5,9,10\}, & \{1,2,4,5,8,10\}, & \{1,2,4,5,7,10\}, \\ \{1,2,4,5,6,10\}, & \{1,2,4,5,6,9\}, & \{1,2,4,5,6,8\}, \\ \{1,2,4,5,6,7\}, & \{1,2,3,7,9,10\}, & \{1,2,3,7,8,10\}, \\ \{1,2,3,7,8,9\}, & \{1,2,3,6,8,10\}, & \{1,2,3,6,8,9\}, \\ \{1,2,3,6,7,10\}, & \{1,2,3,6,7,9\}, & \{1,2,3,6,7,8\}, \\ \{1,2,3,5,7,10\}, & \{1,2,3,5,7,9\}, & \{1,2,3,5,7,8\}, \\ \{1,2,3,5,6,10\}, & \{1,2,3,5,6,9\}, & \{1,2,3,5,6,8\},\end{array}$
$\{1,2,3,5,6,7\}$, $\{1,2,3,4,8,10\}$, $\{1,2,3,4,8,9\}$, $\{1,2,3,4,7,10\}$, $\{1,2,3,4,7,9\}$, $\{1,2,3,4,7,8\}$, $\{1,2,3,4,6,8\}$, $\{1,2,3,4,6,7\}$, $\{1,2,3,4,5,10\}$, $\{1,2,3,4,5,9\}$, $\{1,2,3,4,5,8\}$, $\{1,2,3,4,5,7\}$, $\{1,2,3,4,5,6\}\}$

Therefore, $\mathrm{d}_{t}^{(1)}(P, 6)=84$. i.e, $\mathrm{d}_{\mathrm{t}}(P, 6)=\frac{10}{6} \cdot 84=$ 140

By Lemma 3.5, $\mathrm{d}_{t}(P, 7)=\left(\begin{array}{c}10 \\ 7\end{array}\right)-10=120-10=110$ Again by Lemma 3.4, $\mathrm{d}_{t}(P, 8)=\left(\begin{array}{c}10 \\ 8\end{array}\right)=45, \mathrm{~d}_{t}(P, 9)=$ $\left(\begin{array}{c}10 \\ 9\end{array}\right)=10$ and $\mathrm{d}_{t}(P, 10)=\left(\begin{array}{c}10 \\ 10\end{array}\right)=1$.

Hence, the total domination polynomial of $P$ is $\mathrm{D}_{t}(P$, $x)=10 x^{4}+72 x^{5}+140 x^{6}+110 x^{7}+45 x^{8}+10 x^{9}+x^{10}$.

\section{Theorem 3.7}

Let $G$ be a graph of order $n$ with total domination polynomial $\mathrm{D}_{t}(G, x)=\sum_{i=2}^{n} d_{\mathrm{t}}(G, i) x^{i}$. If $\mathrm{d}_{t}(G, j)=\left(\begin{array}{l}n \\ j\end{array}\right)$ for some $j$, then $\delta(G)>n-j$. Moreover, $\delta(G)=n-(m-$ $1)$, where $m=\min \left\{j / \mathrm{d}_{t}(G, j)=\left(\begin{array}{l}n \\ j\end{array}\right)\right\}$. If for any two vertices of degree $\delta(G)$, say $u$ and $v$ with $\mathrm{N}(u) \neq \mathrm{N}(v)$, then there are exactly $\left(\begin{array}{c}n \\ m-1\end{array}\right)-\mathrm{d}_{t}(G, m-1)$ vertices of degree $\delta(G)$.

\section{Proof :}

Let $\mathrm{G}$ be a simple graph without isolated vertices of order $n$. Let the total domination polynomial of $G$ be $\mathrm{D}_{t}(G, x)=\sum_{i=2}^{n} d_{t}(G, i) x^{i}$. Suppose $\mathrm{d}_{t}(G, j)=\left(\begin{array}{l}n \\ j\end{array}\right)$, then $\mid$ $\mathfrak{D}_{t}(G, j) \mid=\left(\begin{array}{l}n \\ j\end{array}\right)$. This means that any total dominating sets of G with cardinality $j$ can be chosen in $\left(\begin{array}{l}n \\ j\end{array}\right)$ ways, which is same as saying that deleting $n-j$ vertices of $G$ in $\left(\begin{array}{c}n \\ n-j\end{array}\right)$ ways to get the collection of total dominating sets of $G$ with cardinality $j$. Hence, the total dominating sets of $G$ with cardinality $j$ can be constructed by deleting any $n-j$ vertices from the vertex set of $G$. Let $\mathrm{N}(u)$ be the open neighborhood of any vertex $u$ in $G$. If we delete $n-j$ elements from $\mathrm{N}(u)$, then there exists atleast one vertex in $\mathrm{N}(u)$. Otherwise, $u$ becomes an 
isolated vertex. So, each vertex is of degree grater than $n-j$. Hence, $\delta(G)>n-j$. Let $m=\min \left\{j / \mathrm{d}_{t}(G, j)=\left(\begin{array}{l}n \\ j\end{array}\right)\right\}$

Then, $\delta(G)=n-(m-1)=k$ (say). That is, $m-1=n-k$.

Let $u$ and $v$ be any two vertices of degree $k$ in $G$. Then the neighborhoods $N[u]$ and $N[v]$ contain $k+1$ elements of the vertex set of $G$. By the above argument, if we delete $k$ neighboring points of $u$, then $u$ becomes an isolated vertex. Therefore, the remaining set of vertices will not form a total dominating set of cardinality $n-k$. On the other hand, if we delete $k-1$ elements from $N[u]$, except $u$ and one vertex from any other neighborhood, then the remaining vertex set form a total dominating set of cardinality $n-k$. Since, $N[u] \neq$ $N[v]$, there are exactly $n$ non-total dominating sets of $G$. Therefore, $\mathrm{d}_{t}(G, n-k)=\left(\begin{array}{c}n \\ n-k\end{array}\right)-n$. Hence, there are exactly $\left(\begin{array}{l}n \\ j\end{array}\right)-\mathrm{d}_{t}(G, m-1)$ number of vertices of degree $k$.

\section{Remark 3.8}

In Theorem 3.7 the condition $\mathrm{N}(u) \neq \mathrm{N}(v)$ is necessary.

Proof:

For example, we consider a graph $G$ given in the following Figure.

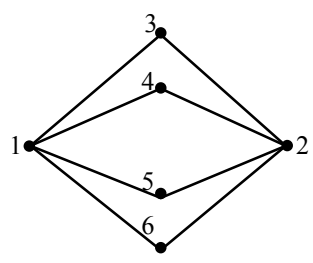

We have, $N(1)=\{3,4,5,6\}$ and $N(2)=\{3,4,5,6\}$. Therefore, $\mathrm{N}(1)=\mathrm{N}(2)$

Since this graph is a complete bipartite graph with partition sets $\mathrm{V}_{1}=\{1,2\}$ and $\mathrm{V}_{2}=\{3,4,5,6\}$, the total domination polynomial of the graph $\mathrm{G}$ is

$$
\begin{aligned}
& \mathrm{D}_{t}(G, x)=\left[(1+x)^{2}-1\right]\left[(1+x)^{4}-1\right] \\
& =x^{6}+6 x^{5}+14 x^{4}+16 x^{3}+8 x^{2} .
\end{aligned}
$$

For this graph, $m=5$ and $\delta(G)=2$ and we have 4 vertices of degree 2 .

But, by Theorem 3.7, we must have $\left(\begin{array}{l}6 \\ 4\end{array}\right)-\mathrm{d}_{t}(G, 4)=$ $15-14=1$ is the number of vertices of degree $\delta(G)$, which is not true.

\section{Theorem 3.9}

Let $\mathrm{H}$ be a $k$ - regular graph, where for any two vertices $u, v \in \mathrm{V}(H), \mathrm{N}(u) \neq \mathrm{N}(v)$. If $\mathrm{D}_{t}(G, x)=\mathrm{D}_{t}(H$, $x)$, then $G$ is also a $k$ - regular graph.

\section{Proof:}

Let $H$ be a $k$ - regular graph of order $n$. The total domination polynomial of $H$ is $\mathrm{D}_{t}(H, x)=\sum_{i=2}^{n} d_{t}(H, i) x^{i}$. Since $\mathrm{D}_{t}(G, x)=\mathrm{D}_{t}(H, x), \mathrm{D}_{t}(G, x)=\sum_{i=2}^{n} d_{t}(H, i) x^{i}$. Since $H$ is $k$ - regular graph, $\mathrm{d}_{t}(G, j)=\left(\begin{array}{l}n \\ j\end{array}\right)$ for all $j>n$ - $k$. Since $\mathrm{N}(u) \neq \mathrm{N}(v)$ and by Theorem 3.7, there are exactly $n$ vertices of degree $k$. Hence, $G$ is a $k$ - regular graph.

\section{References}

[1] Akbari, S., Alikhani, S. and Peng, Y.H. 2010, Characterization of graphs using Domination Polynomial, European Journal of Combinatorics, Volume 31, Issue 7, October 2010, Pages 1714 - 1724.

[2] Alikhani.S and Hamzeh Torabi, 2010, On Domination Polynomials of complete partite Graphs, World Applied Sciences Journal, 9(1):23-24.

[3] Alikhani.S and Peng.Y.H, 2009, Introduction to Domination Polynomial of a graph, arXiv:0905.2251v1 [math.CO] 14 May.

[4] Alikhani. S. and Peng, Y.H, 2010, Dominating sets and Domination Polynomials of Certain Graphs, H, Opuscula Mathematica, Volume 30, No 1, Pages 37 - 51.

[5] Alikhani. S, Yee-Hock Peng, 2011, Domination Polynomials of Cubic graphs of order 10, Turkish Journal of Mathematics, Volume 35, Issue 3, Pages 335 - 366.

[6] Chartrand.G and Zhang.P, 2004, Introduction to Graph Theory, Mc Graw Hill, Higher Education India August ISBN: 978007123829.

[7] Vijayan.A, Sanal Kumar.S, 2012, On Total Domination Polynomial of graphs, Global Journal of Theoretical and Applied Mathematical Sciences, ISSN 2248-9916, Volume 2, Issue 2, Pages. 91-97. 